\title{
Doing cell biology in embryos: regulated membrane traffic and its implications for cadherin biology
}

\section{Aparna Ratheesh and Alpha S Yap*}

Address: Institute for Molecular Bioscience and Division of Molecular Cell Biology, The University of Queensland, St Lucia, Brisbane, Queensland 4072, Australia

* Corresponding author: Alpha S Yap (a.yap@uq.edu.au)

Fl000 Biology Reports 2010, 2:30 (doi:10.34I0/B2-30)

The electronic version of this article is the complete one and can be found at: http://fl000.com/reports/biology/content/2/30

\begin{abstract}
Regulated trafficking of cadherin adhesion molecules is often invoked as a mechanism to generate dynamic adhesive cell-cell contacts for tissue modeling and morphogenesis. The past 2-3 years have seen several important papers that tackle the cell biology of cadherin trafficking in organismal systems to provide new insights into both mechanism and morphogenetic impact.
\end{abstract}

\section{Introduction and context}

Adhesive interactions between cells and their environment (other cells and the extracellular matrix) are key regulators of tissue organization. In the past several years, we have come to appreciate that these interactions are intrinsically dynamic, and it has been attractive to postulate that adhesion dynamics may contribute to the remodeling of cell-cell and cell-matrix interactions associated with tissue morphogenesis. In particular, membrane traffic of adhesion molecules has been intensively studied as a cellular mechanism for dynamic turnover of both adhesive contacts and cellular interactions. An important recent advance has been the application of organismal systems (notably Drosophila and zebrafish) to study adhesion receptor trafficking. These efforts to 'do cell biology in organisms' carry the advantage of allowing genetic approaches to be used to identify new molecular regulators of receptor trafficking. They also provide the opportunity to test the potential morphogenetic impact of these cellular processes. Although a range of adhesion receptors undergo regulated trafficking, we focus here on recent studies that characterized the molecular regulation of cadherin transport and its functional implications in a variety of developmental systems.

\section{Major recent advances \\ Characterizing cadherin trafficking and its molecular regulation}

Cadherin trafficking and its molecular characterization were originally performed using a variety of cultured mammalian epithelial cells (reviewed in $[1,2]$ ). An important stimulus in this field was the demonstration that E-cadherin undergoes endocytic recycling in cultured MDCK epithelial cells [3]. It is now clear that this pathway operates also in Drosophila and zebrafish. For example, the Peifer lab [4] recently demonstrated that DE-cadherin in the embryonic ectoderm and amnioserosa is trafficked through both Rab5- and Rab11-positive endosomes, as is also observed in mammalian cells $[3,5,6]$. Moreover, recycling can be affected at several points in the trafficking itinerary to perturb adherens junction integrity. Disruption of the early endosomal regulator Rab5 as well as Rab11, the latter of which controls traffic through recycling endosomes, both perturbed cadherin junction integrity [4]. Furthermore, cells in the pupal notum from Drosophila embryos mutant for exocyst components accumulated DE-cadherin in recycling endosomes and displayed abnormal adherens junctions $[7,8]$. This implies that the integrity of adherens junctions in these developing tissues 
depends on a continuous flux of DE-cadherin through a membrane recycling pathway. Interestingly, the cortical localization of the exocyst in the Drosophila retina has itself been observed to depend on the integrity of adherens junctions [9]. This suggests that what we perceive as junctional integrity may arise as an emergent property from positive feedback between membrane recycling, exocyst localization, and junctions themselves.

The genetic amenability of Drosophila has also been instrumental in identifying new regulators of cadherin trafficking. Recently, the laboratories of Buzz Baum [10] and Yohann Bellaiche [11] independently identified a novel impact of Cdc42 on DE-cadherin trafficking. Both groups reported that loss of Cdc42 function perturbed adherens junction integrity and also blocked endocytosis of cadherin. Cdc42 was genetically linked to signaling through the Par6/atypical protein kinase C (PKC) pathway, thereby identifying a link between cadherin traffic and a well-described regulator of epithelial polarity. Furthermore, both groups defined downstream genetic interactions with the cytoskeletal regulators WASP and Arp2/3, as well as with dynamin itself $[10,11]$. This suggested that the Cdc42/Par6/aPKC pathway promotes cadherin internalization, perhaps by regulating the molecular machinery responsible for scission of endocytic precursors to generate vesicles [11]. Consistent with this, Warner and Longmore [12] reported that depletion of Rho in the fly eye increased DE-cadherin internalization in a Cdc42-dependent manner.

One tacit attraction of studying organismal systems is the hope that development in the embryo might provide a robustness that avoids the variability that can be associated with experiments that use cultured cell systems. However, even here, the complexity of biology reminds us that this is not necessarily so. This is exemplified by another recent study in which Harris and Tepass [13] also demonstrate a genetic requirement for Cdc42 to stabilize adherens junctions in the dynamic remodeling tissue of the ventral neuroectoderm. However, they show that here Cdc42 serves to inhibit, rather than promote, endocytosis and recycling of apical membrane components, such as Crumbs and Patj. Furthermore, they provide evidence that the junctional phenotype may be a secondary effect of disrupting the apical localization of Crumbs, which is a known regulator of junctional integrity [14]. This reminds us that there are limits to the extent to which molecular mechanism can be inferred from genetic interactions. Finally, they demonstrate that Cdc42 is preferentially required in the most dynamic tissues, emphasizing that biological context may condition the impact of these signaling pathways. Similarly, Roeth et al. [4] showed that the morphogenetic impact of disrupting Rab5 or Rab11 is also tissue-specific.

\section{Morphogenetic impacts of cadherin trafficking}

The identification of key regulators of cadherin traffic provides the convenient opportunity to test their morphogenetic impact in developing embryos, beyond their consequences for adherens junction integrity. This is illustrated during zebrafish gastrulation, in which formation of the prechordal plate requires that its progenitors migrate as a cohesive group of cells from the blastoderm margin in a process that depends on the planar polarity signal, Wnt11. Strikingly, Ulrich et al. [15] demonstrated that blocking Rab5C function with antisense morpholinos prevented Wnt11-induced prechordal plate formation. Interestingly, Wnt11 also induces E-cadherin internalization through a Rab5c-dependent pathway. This suggests that induced cadherin internalization might contribute to morphogenetic cellular rearrangements in response to Wnt11.

Cadherin recycling has also been implicated in a variety of tissue rearrangements in the fly. In the Drosophila wing, epithelial cells undergo a striking rearrangement during development, being irregularly packed during the larval and prepupal stages, then becoming reorganized into hexagonal arrays shortly before hair formation occurs. Interestingly, E-cadherin is actively endocytosed and recycled through Rab11 endosomes during the process of hexagonal rearrangement. Disruption of endocytosis with dynamin mutants blocks this cellular reorganization [16], suggesting that cadherin recycling may contribute to turnover of junctions necessary for cell-upon-cell rearrangements. Again, however, tissue and cellular context can significantly affect the impact of membrane traffic on cellular rearrangement. Another form of cell-cell movement is cellular intercalation, which has been implicated in tissue elongation events during development [17]. However, in contrast to the Drosophila wing, Rab11mediated recycling appeared to inhibit cellular intercalation in the dorsal trunk of the tracheal system [18]. This implies that membrane trafficking may have quite different morphogenetic impacts depending on the type of morphogenetic movement involved and the tissues being studied.

\section{Future directions}

We have focused here on how recent analysis of organismal models has provided new insights into both the molecular regulation and morphogenetic impact of cadherin trafficking. In the latter case, we have also concentrated on cellular rearrangements, but it is likely that cadherin trafficking will have increasingly wideranging consequences. For instance, during Drosophila 
oogenesis, germline stem cells (GSCs) are attached to anterior niche cap cells via adherens junctions and this physical attachment helps to maintain the balance between self-renewing and differentiating cells. Rab11 regulates the connections between the GSCs and the niche cells through polarized trafficking of E-cadherin and other apical proteins [19], implying that Rab11 could indirectly affect self-renewal and proliferation in the stem cell niche.

Permit us to finish, though, with a challenge to the field. Ultimately, detailed progress in understanding the biological impact of cadherin trafficking will require tools that selectively perturb cadherin traffic. To date, the functional impact of cadherin trafficking has been analyzed using techniques that target key regulators of membrane traffic generally, but not necessarily cadherin traffic specifically. Although these have yielded exciting results, such as those reviewed here, there is a very real possibility that some changes in cadherin biology result as indirect effects of perturbing other trafficking events, such as apical localization of Crumbs [13]. For example, clathrin and Rab5 are reported to control cortical signaling of Rac [20], which can affect E-cadherin adhesion and junctional organization $[21,22]$. And dynamin, best understood for its impact on membrane trafficking, was recently identified as regulating global acto-myosin contractility [23], which also supports junctional integrity [24]. Ongoing efforts to elucidate specific regulation of cadherin trafficking, such as recent efforts to understand the mechanism of cadherin internalization [25], will then be essential if we are to specifically ablate cadherin trafficking. The combination of those tools, combined with the range of organismal systems now available to test their impact, will portend heady times in our efforts to understand the cellular basis of morphogenesis.

\section{Abbreviations}

GSC, germline stem cell; MDCK, Madin-Darby canine kidney; PKC, protein kinase C; WASP, Wiskott-Aldrich syndrome protein.

\section{Competing interests}

The authors declare that they have no competing interests.

\section{Acknowledgments}

The authors were funded by the Human Frontiers Science Program and the National Health and Medical Research Council of Australia.

\section{References}

I. Bryant DM, Stow JL: The ins and outs of E-cadherin trafficking. Trends Cell Biol 2004, 14:427-34.

2. Delva E, Kowalczyk AP: Regulation of cadherin trafficking. Traffic 2009, 10:259-67.
3. Le TL, Yap AS, Stow JL: Recycling of E-cadherin: a potential mechanism for regulating cadherin dynamics. J Cell Biol 1999, 146:219-32.

4. Roeth JF, Sawyer JK, Wilner DA, Peifer M: Rab I I helps maintain apical crumbs and adherens junctions in the Drosophila embryonic ectoderm. PLoS One 2009, 4:e7634.

5. Lock JG, Stow JL: Rab II in recycling endosomes regulates the sorting and basolateral transport of E-cadherin. Mol Biol Cell 2005, 16:1744-55.

6. Desclozeaux M, Venturato J, Wylie FG, Kay JG, Joseph SR, Le HT, Stow JL: Active Rab I I and functional recycling endosome are required for E-cadherin trafficking and lumen formation during epithelial morphogenesis. Am J Physiol Cell Physiol 2008, 295:C545-56.

7. Blankenship JT, Fuller MT, Zallen JA: The Drosophila homolog of the Exo84 exocyst subunit promotes apical epithelial identity. J Cell Sci 2007, I20:3099-II0.

8. Langevin J, Morgan MJ, Sibarita JB, Aresta S, Murthy M, Schwarz T, Camonis J, Bellaiche Y: Drosophila exocyst components Sec5, Sec6, and Secl5 regulate DE-Cadherin trafficking from recycling endosomes to the plasma membrane. Dev Cell 2005, 9:365-76.

FI000 Factor 4.8 Must Read

Evaluated by Jean-Paul Vincent 06 Oct 2005, Michel Labouesse 14 Nov 2005

9. Beronja S, Laprise P, Papoulas O, Pellikka M, Sisson J, Tepass U: Essential function of Drosophila Sec6 in apical exocytosis of epithelial photoreceptor cells. J Cell Biol 2005, 169:635-46.

FI000 Factor 6.0 Must Read

Evaluated by Michel Labouesse II Nov 2005

10. Georgiou M, Marinari E, Burden J, Baum B: Cdc42, Par6, and aPKC regulate Arp2/3-mediated endocytosis to control local adherens junction stability. Curr Biol 2008, 18:1631-8.

II. Leibfried A, Fricke R, Morgan MJ, Bogdan S, Bellaiche Y: Drosophila Cip4 and WASp define a branch of the Cdc42-Par6-aPKC pathway regulating E-cadherin endocytosis. Curr Biol 2008, 18:1639-48.

12. Warner SJ, Longmore GD: Distinct functions for Rhol in maintaining adherens junctions and apical tension in remodeling epithelia. J Cell Biol 2009, I85: I I I-25.

13. Harris KP, Tepass U: Cdc42 and Par proteins stabilize dynamic adherens junctions in the Drosophila neuroectoderm through regulation of apical endocytosis. J Cell Biol 2008, 183: 1129-43.

14. Knust $\mathrm{E}$, Bossinger $\mathrm{O}$ : Composition and formation of intercellular junctions in epithelial cells. Science 2002, 298: 1955-9.

15. Ulrich F, Krieg M, Schotz EM, Link V, Castanon I, Schnabel V, Taubenberger A, Mueller D, Puech PH, Heisenberg CP: WntII functions in gastrulation by controlling cell cohesion through Rab5c and E-cadherin. Dev Cell 2005, 9:555-64.

FI000 Factor 6.7 Must Read

Evaluated by Lilianna Solnica-Krezel 28 Nov 2005, Adam Linstedt 07 Oct 2005, Jim Smith 07 Oct 2005, Erez Raz 24 Oct 2005, Anna Huttenlocher 26 Oct 2005

16. Classen AK, Anderson KI, Marois E, Eaton S: Hexagonal packing of Drosophila wing epithelial cells by the planar cell polarity pathway. Dev Cell 2005, 9:805-17.

FI000 Factor 6.4 Must Read

Evaluated by Ken Irvine I4 Dec 2005, Thomas Lecuit 25 Jan 2006

17. Pilot F, Lecuit $\mathrm{T}$ : Compartmentalized morphogenesis in epithelia: from cell to tissue shape. Dev Dyn 2005, 232:685-94. 
18. Shaye DD, Casanova J, Llimargas M: Modulation of intracellular trafficking regulates cell intercalation in the Drosophila trachea. Nat Cell Biol 2008, 10:964-70.

FI000 Factor 6.0 Must Read

Evaluated by Michel Labouesse 28 Jul 2008

19. Bogard N, Lan L, Xu J, Cohen RS: Rab I I maintains connections between germline stem cells and niche cells in the Drosophila ovary. Development 2007, 134:3413-8.

20. Palamidessi A, Frittoli E, Garre M, Faretta M, Mione M, Testa I, Diaspro A, Lanzetti L, Scita G, Di Fiore PP: Endocytic trafficking of Rac is required for the spatial restriction of signaling in cell migration. Cell 2008, I34:135-47.

FI000 Factor 6.8 Must Read

Evaluated by Harald Stenmark 18 Aug 2008, Christopher Marshall 23 Jul 2008, Marie-France Carlier 3 I Jul 2008, Gary Bokoch 27 Nov 2008

21. Goodwin M, Kovacs EM, Thoreson MA, Reynolds AB, Yap AS: Minimal mutation of the cytoplasmic tail inhibits the ability of
E-cadherin to activate Rac but not phosphatidylinositol 3-kinase: direct evidence of a role for cadherin-activated Rac signaling in adhesion and contact formation. J Biol Chem 2003, 278:20533-9.

22. Takaishi K, Sasaki T, Kotani H, Nishioka H, Takai Y: Regulation of cell-cell adhesion by Rac and Rho small G proteins in MDCK cells. J Cell Biol 1997, I39:1047-59.

23. Chua J, Rikhy R, Lippincott-Schwartz J: Dynamin 2 orchestrates the global actomyosin cytoskeleton for epithelial maintenance and apical constriction. Proc Natl Acad Sci U S A 2009, [Epub ahead of print].

24. Shewan AM, Maddugoda M, Kraemer A, Stehbens SJ, Verma S, Kovacs EM, Yap AS: Myosin 2 is a key Rho kinase target necessary for the local concentration of E-cadherin at cellcell contacts. Mol Biol Cell 2005, 16:453 I-2.

25. Chiasson CM, Wittich KB, Vincent PA, Faundez V, Kowalczyk AP: pI20-catenin inhibits VE-cadherin internalization through a Rho-independent mechanism. Mol Biol Cell 2009, 20:1970-80. 